\title{
Genotypic Heterogeneity of Orientia tsutsugamushi in Scrub Typhus Patients and Thrombocytopenia Syndrome Co-infection, Myanmar
}

Aye Marlar Win, ${ }^{1}$ Yen Thi Hai Nguyen, ${ }^{1}$ Yuri Kim, ${ }^{1}$ Na-Young Ha, Jun-Gu Kang, Hongil Kim, Bo San, Okkar Kyaw, Wah Win Htike, Dong-Ok Choi, Keun-Hwa Lee, ${ }^{2} \mathrm{Nam}-\mathrm{Hyuk} \mathrm{Cho}^{2}$

Serologic and molecular surveillance of serum collected from 152 suspected scrub typhus patients in Myanmar revealed Orientia tsutsugamushi of genotypic heterogeneity. In addition, potential co-infection with severe fever with thrombocytopenia syndrome virus was observed in 5 (3.3\%) patients. Both scrub typhus and severe fever with thrombocytopenia syndrome are endemic in Myanmar.

Scrub typhus is a miteborne febrile illness caused by the bacterium Orientia tsutsugamushi, which is endemic in the Asia-Pacific region and a major cause of undifferentiated febrile disease (1). O. tsutsugamushi infections were documented in Myanmar during the 1940s (2). Since then, however, no report has described the prevalence and genetics of scrub typhus in Myanmar, although 2 studies, including 1 from 2017, identified scrub typhus as one of the primary infections causing acute febrile illness on the Thailand-Myanmar border $(3,4)$. These results underscore the need for research on this vectorborne infection in Myanmar, including studies defining the genotypic diversity of $O$. tsutsugamushi. Lack of this information has been a serious obstacle to developing effective diagnostic methods and a vaccine for scrub typhus (1).

The tickborne virus severe fever with thrombocytopenia syndrome virus (SFTSV), of the genus

Author affiliations: University of Medicine 1, Yangon, Myanmar (A.M. Win, O. Kyaw, W.W. Htike); Seoul National University College of Medicine, Seoul, South Korea (Y.T.H. Nguyen, Y. Kim, N.-Y. Ha, J.-G. Kang, H. Kim, N.-H. Cho); Private practitioner, Sagaing, Myanmar (B. San); Bore Da Biotech, Seongnam, South Korea (D.-O. Choi); Hanyang University College of Medicine, Seoul (K.-H. Lee); Seoul National University Medical Research Center and Bundang Hospital, Seoul (N.-H. Cho)

DOI: https://doi.org/10.3201/eid2608.200135
Banyangvirus, can cause hemorrhagic fever with a mortality rate of up to $40 \%$ (5). SFTSV infections are endemic in eastern Asia, and retrospective studies have confirmed its presence in China in 1996 (6), South Korea in 2000 (7), Japan in 2005 (8), and Vietnam in 2017 (9). In addition, mixed infection with SFTSV and $O$. tsutsugamushi has been detected in patients in South Korea, where both pathogens are endemic $(7,10)$. These results further emphasize the urgent need for epidemiologic studies of vectorborne diseases in areas of endemicity to improve our ability to accurately differentiate febrile infectious diseases with atypical signs and symptoms during the initial stages so they can be promptly treated. Here, we used blood samples from suspected scrub typhus patients in Myanmar to investigate the serologic prevalence and genotypic diversity of $O$. tsutsugamushi. We also examined these patients for possible co-infection with SFTSV, which has been an emerging threat to public health in eastern Asia.

\section{The Study}

To investigate the genotypic diversity of O.tsutsugamushi and potential co-infection with SFTSV in Myanmar, we collected whole blood samples from 152 clinically suspected scrub typhus patients (Table; Appendix Table 1, https://wwwnc.cdc.gov/EID/ article/26/8/20-0135-App1.pdf) in Sagaing and Magway Provinces (Figure 1) during February 2018-January 2019. Mean age of the suspected scrub typhus patients was $27 \pm 19.8$ years (range of $2-73$ years). We observed eschar, a selection criteria for scrub typhus, in $144(94.7 \%)$ of the 152 patients. Mean fever duration was 6 days ( $\mathrm{SD} \pm 2.9$ days).

\footnotetext{
${ }^{1}$ These first authors contributed equally to this article.

${ }^{2}$ These authors were co-principal investigators.
} 
Table. Baseline characteristics and summary of serologic and molecular diagnosis of suspected scrub typhus patients enrolled in study of genotypic heterogeneity of Orientia tsutsugamushi, Myanmar

\begin{tabular}{lc}
\hline Category & Value \\
\hline Age & \\
Age, y mean \pm SD & $27.0 \pm 19.8$ \\
Age distribution, y & \\
$\leq 10$ & $38(25.0)$ \\
$11-20$ & $38(25.0)$ \\
$21-30$ & $23(15.1)$ \\
$31-40$ & $10(6.6)$ \\
$41-50$ & $17(11.0)$ \\
$51-60$ & $16(10.5)$ \\
$\geq 61$ & $10(6.6)$ \\
\hline Sex ratio, M:F $\%$ male) & $93 / 59(61.2)$ \\
\hline Clinical variables & \\
Fever duration, d, mean $\pm S D$ & $6.1 \pm 2.9$ \\
Eschar & $144(94.7)$ \\
Rash & $3(2.0)$ \\
Myalgia & $25(20)$ \\
\hline Method of diagnosis of scrub typhus & \\
ICT & $41 / 128(32.0)$ \\
TSA56 IgG & $36 / 128(28.1)$ \\
ScaA IgG & $25 / 128(19.5)$ \\
IFA & $138 / 152(90.8)$ \\
O. tsutsugamushi IgG & $119 / 152(78.3)$ \\
O. tsutsugamushi IgM & $90 / 152(59.2)$ \\
PCR (tsa56) & $9 / 152(5.9)$ \\
\hline Method of diagnosis of SFTSV & \\
RT-PCR & $5 / 152(3.3)$ \\
\hline
\end{tabular}

*Values are no. (\%) patients or no. patients/no. tested (\%) except as indicated. ICT, immunochromatography test; IFA, indirect

immunofluorescence assay; RT-PCR, reverse transcription PCR; SFTSV, severe fever with thrombosis syndrome virus.

For initial serologic diagnosis of 128 serum samples, we used immunochromatography test strips coated with TSA56 and ScaA antigens, which revealed respective positive rates of $28.1 \%(36 / 128)$ and $19.5 \%$ (25/128); the overall positive rate was $32.0 \%(41 / 128)$. Of the 128 samples, $20(15.6 \%)$ reacted with both antigens and 5 of $36(13.9 \%)$ were positive for ScaA antigen only (Table; Appendix Figure 1), suggesting a potential applicability of ScaA antigen, when used simultaneously with TSA56 antigen, for the diagnosis of scrub typhus during the acute phase (11). To confirm serologic positivity against the bacterial antigen, we also conducted an indirect immunofluorescence assay using cells infected with $O$. tsutsugamushi, the standard method for diagnosing scrub typhus (12). Among the 152 serum samples we tested, results were positive $(\geq 1: 40)$ for $119(78.3 \%)$ for specific IgG and for 90 (59.2\%) for IgM (Table; Appendix Table 1). Median titers of the positive serum samples were 1:640 for both IgG and IgM. Among the suspected scrub typhus patients, test results for $13(8.6 \%)$ serum samples were negative for both IgG and IgM against O. tsutsugamushi.

For molecular diagnosis of scrub typhus, we examined all the serum samples by PCR to confirm infection and identify the genotypes of $O$. tsutsugamushi in the patients in Myanmar. From the 152 serum samples, we detected specific PCR products in 9 (5.9\%) and sequenced them for genotyping. We compared results of phylogenetic analysis of the 9 tsa56 gene sequences with sequences from 17 protogenotypes (1), which revealed $\geq 5$ genotypes, including Karp_A (4/9, $44.4 \%)$, Karp_B (1/9, 11.1\%), Kato_B (2/9, 22.2\%), Gilliam $(1 / 9,11.1 \%)$, and JG_C $(1 / 9,11.1 \%)$ (Figure 2).

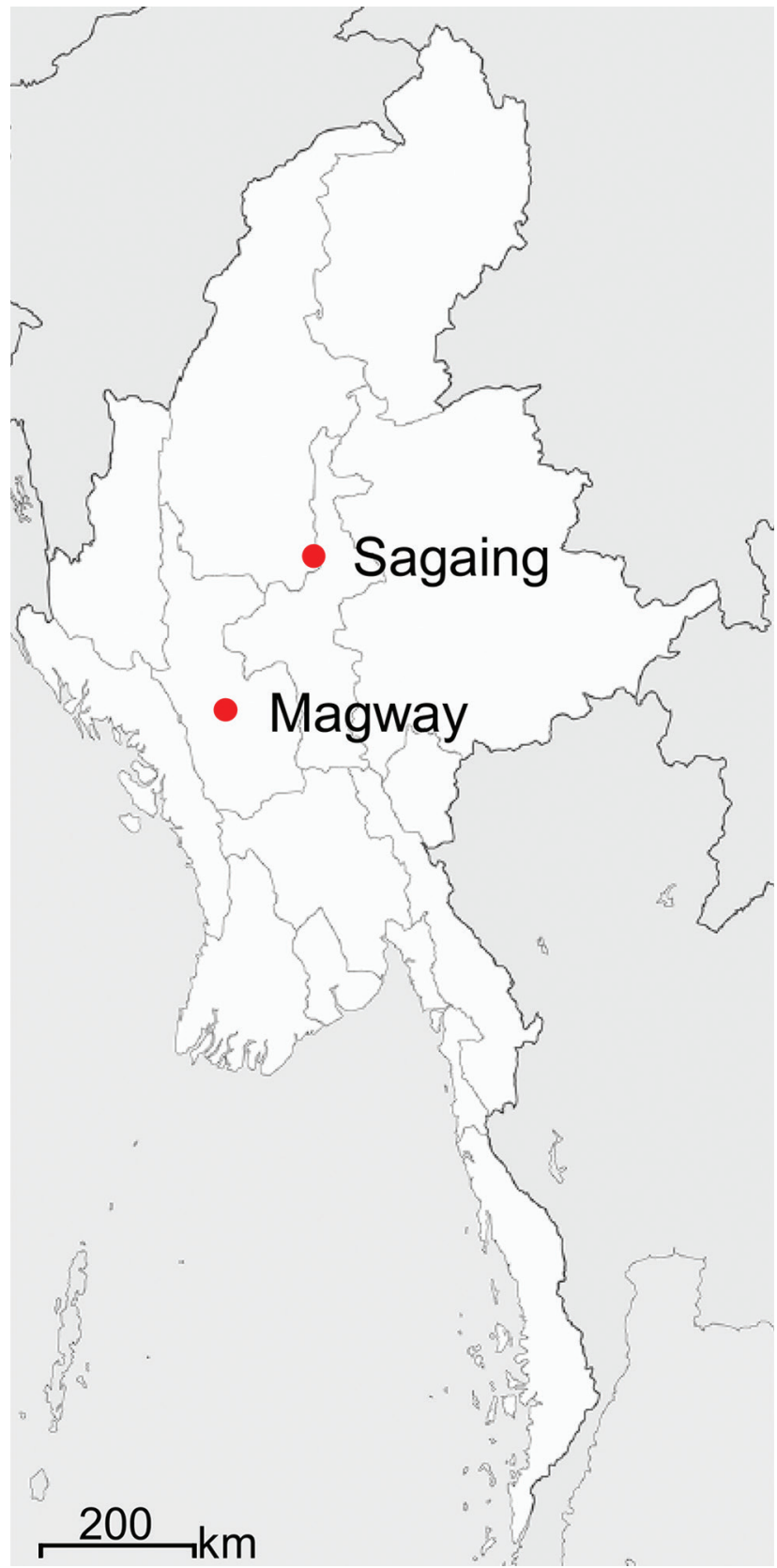

Figure 1. Locations in Sagaing and Magway Provinces in Myanmar, where suspected scrub typhus patients' serum samples were collected for study of genotypic heterogeneity of Orientia tsutsugamushi. 


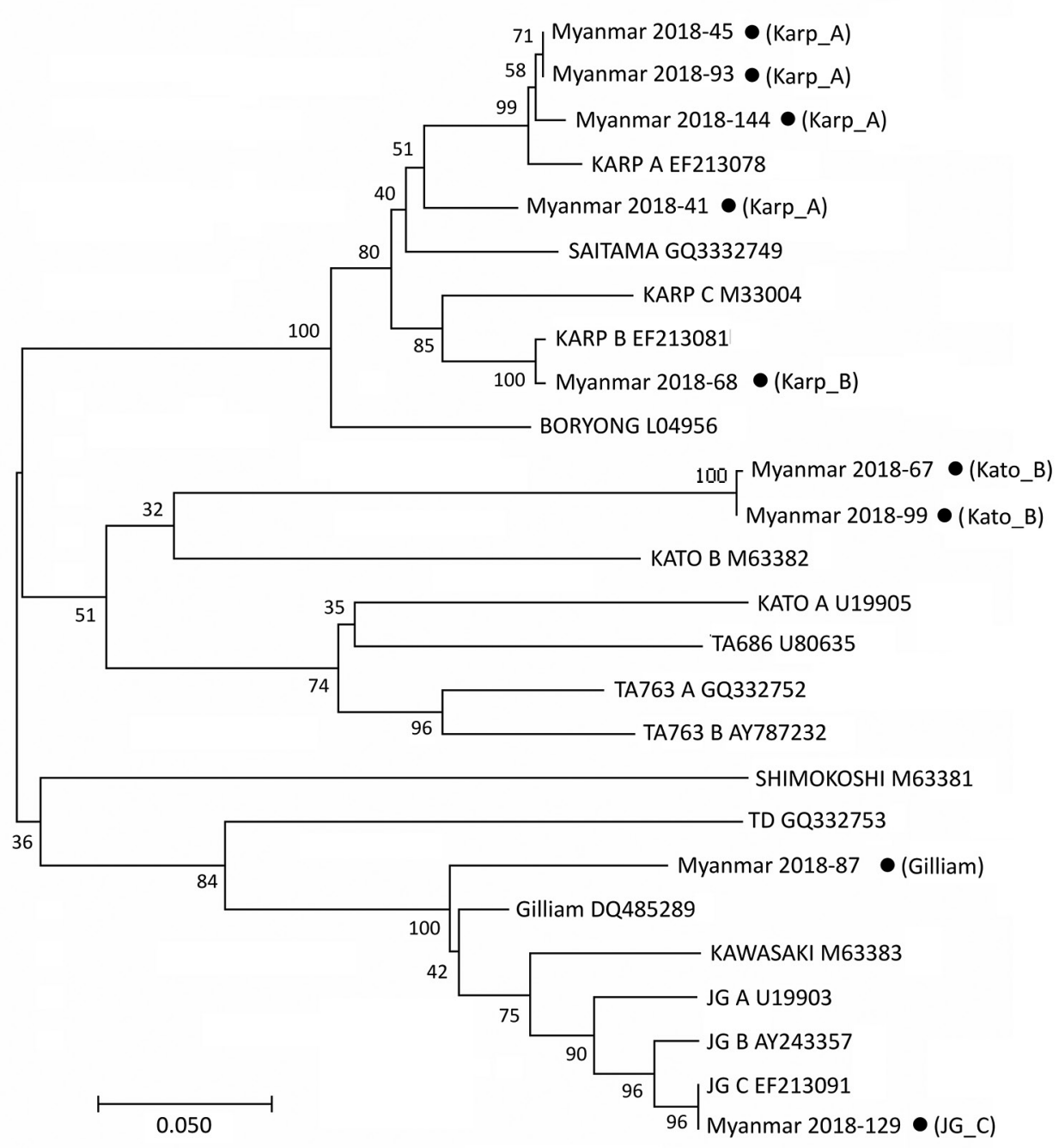

Figure 2. Phylogenetic tree constructed on the basis of Orientia tsutsugamushi tsa56 gene sequences for scrub typhus patients in Myanmar (black dots) and reference sequences. The tree was constructed using the maximum likelihood method with MEGA7 (http://www.megasoftware.net). The tsa56 gene sequences identified in this study are indicated by black circles and compared with 17 representative genotype sequences reported by a previous study (1). The percentage of replicate trees in which the associated genotypes clustered together in the bootstrap test (1,000 replicates) is shown next to the branches.

Finally, we used reverse transcription PCR analysis to investigate possible SFTSV infection in the patients $(7,9,13)$. Among 152 patients' serum samples, $5(3.3 \%)$ were positive for the partial small (S) segment of the SFTSV RNA genome, indicating SFTSV infection. Results from phylogenetic analysis of the partial $S$ segment sequences showed that 4 isolates were the same as those previously reported from Vietnam (9); 1 isolate differed by 1 base from the other 4 isolates (Appendix Figure 2), suggesting genetic homogeneity of SFTSV in southern Asia. Of note, 4 out of the 5 SFTSV-positive patients had eschar, and 4 were $<15$ years of age (Appendix Figure $2)$. Furthermore, 3 of them carried high titers $(\geq 1$ : 2560) of IgG, IgM, or both specific to O. tsutsugamushi, as measured by indirect immunofluorescence assay (Appendix Table 1), suggesting co-infection with scrub typhus. All patients were successfully treated and recovered, including the SFTSV-positive febrile patients, after 5-7 days of fever.

\section{Conclusions}

We observed a high prevalence of antibodies against $O$. tsutsugamushi in suspected scrub typhus patients in Myanmar, suggesting that scrub typhus, previously reported in the 1940s, remains prevalent in this country (2). Of note, a high prevalence of scrub typhus in children was confirmed (4); therefore, young children with febrile illness should be carefully observed for early diagnosis and treatment of scrub typhus. Because we were only able to examine serum samples collected from patients during the acute phase of infection and could not assess the rise of antibody titers in paired samples collected in convalescent phases, we were not able to confirm the exact rate of prevalence of scrub typhus in the suspected patients. The baseline levels of antibody titers against O. tsutsugamushi in healthy persons need to be assessed to determine the cutoff titer levels for diagnosing acute scrub typhus in the endemic region (14). 
In addition, genotyping $O$. tsutsugamushi revealed that $\geq 5$ different genotypes are currently present and showed genetic heterogeneity in Myanmar. Moreover, we detected possible co-infection with SFTSV and O. tsutsugamushi in 5 patients. None of these patients had a history of travel abroad, and all live in the same village in Sagaing Province, suggesting that there may be hot spots for SFTSV infection. Co-infection with O. tsutsugamushi and SFTSV might be mediated by either simultaneous transmission from 2 different vectors each carrying 1 pathogen or by a single tick or mite species carrying both pathogens (10). Four of 5 SFTSV-positive patients were $<15$ years of age, and all 5 recovered within a week. Given that the disease severity of SFTS is associated with host age and the viral genotype (15), milder clinical symptoms observed in these patients might have been because of exposure at a younger age or prevalence of less virulent genotypes of SFTSV in Myanmar. Therefore, continuous surveillance of SFTS patients needs to be conducted, reporting detailed clinical manifestations and associated viral genotypes prevalent in the local area. In addition, more reliable differential diagnosis techniques and prevention and control measures are required for better clinical practices and outcomes in the endemic regions of multiple tickborne and miteborne pathogens.

This work was supported by a grant from the National Research Foundation of Korea (grant number: 2017M3A9E4061998) and the Korean Health Technology R\&D Project of the Ministry of Health \& Welfare, Republic of Korea (grant number: HI15C2891). N.T.H.Y., Y.K., N.Y.H., J.G.K., and H.K. received a scholarship from the BK21-plus education program provided by the National Research Foundation of Korea.

\section{About the Author}

Dr. Win is a medical microbiologist working at the University of Medicine 1, Yangon, Myanmar. Her primary research interests are epidemiology and pathogenesis of various infectious diseases endemic in Myanmar.

\section{References}

1. Kim G, Ha NY, Min CK, Kim HI, Yen NTH, Lee KH, et al. Diversification of Orientia tsutsugamushi genotypes by intragenic recombination and their potential expansion in endemic areas. PLOS Neglect Trop Dis. 2017;11:e0005408. PubMed https:// doi.org/10.1371/journal.pntd.0005408

2. Davis GE, Austrian RC, Bell EJ. Observations on tsutsugamushi disease (scrub typhus) in Assam and Burma: the recovery of strains of Rickettsia orientalis. Am J Hyg. 1947;46:268-86. https:/ / doi.org/10.1093/oxfordjournals.aje.a119168

3. Parola P, Miller RS, McDaniel P, Telford SR III, Rolain JM, Wongsrichanalai $\mathrm{C}$, et al. Emerging rickettsioses of the Thai-Myanmar border. Emerg Infect Dis. 2003;9:592-5. https://doi.org/10.3201/eid0905.020511
4. Brummaier T, Kittitrakul C, Choovichian V, Lawpoolsri S, Namaik-Larp C, Wattanagoon Y. Clinical manifestations and treatment outcomes of scrub typhus in a rural health care facility on the Thailand-Myanmar border. J Infect Dev Ctries. 2017;11:407-13. https://doi.org/10.3855/jidc.8912

5. Liu S, Chai C, Wang C, Amer S, Lv H, He H, et al. Systematic review of severe fever with thrombocytopenia syndrome: virology, epidemiology, and clinical characteristics. Rev Med Virol. 2014;24:90-102. https:// doi.org/10.1002/rmv.1776

6. Hu J, Shi C, Li Z, Guo X, Qian Y, Tan W, et al. A cluster of cases of severe fever with thrombocytopenia syndrome bunyavirus infection in China, 1996: a retrospective serological study. PLoS Negl Trop Dis. 2018;12:e0006603. https:// doi.org/10.1371/journal.pntd.0006603

7. Thi Hai Yen N, Kim C, Jeong S, Jeon K, Choi H, Ro HJ, et al. Severe fever with thrombocytopenia syndrome virus infection or mixed infection with scrub typhus in South Korea in 2000-2003. Am J Trop Med Hyg. 2019;101:1096-9. https:// doi.org/10.4269/ajtmh.19-0392

8. Takahashi T, Maeda K, Suzuki T, Ishido A, Shigeoka T, Tominaga $\mathrm{T}$, et al. The first identification and retrospective study of severe fever with thrombocytopenia syndrome in Japan. J Infect Dis. 2014;209:816-27. https:/ / doi.org/ 10.1093/infdis/jit603

9. Tran XC, Yun Y, Van An L, Kim SH, Thao NTP, Man PKC, et al. Endemic severe fever with thrombocytopenia syndrome, Vietnam. Emerg Infect Dis. 2019;25:1029-31. https://doi.org/10.3201/eid2505.181463

10. Ra SH, Kim JY, Cha HH, Kwon JS, Lee HJ, Jeon NY, et al. Coinfection of severe fever with thrombocytopenia syndrome and scrub typhus in patients with tick-borne illness. Am J Trop Med Hyg. 2019;101:1259-62. https://doi.org/ 10.4269/ajtmh.19-0242

11. Watthanaworawit $W$, Turner $P$, Turner $C$, Tanganuchitcharnchai A, Jintaworn S, Hanboonkunupakarn B, et al. Diagnostic accuracy assessment of immunochromatographic tests for the rapid detection of antibodies against Orientia tsutsugamushi using paired acute and convalescent specimens. Am J Trop Med Hyg. 2015;93:1168-71. https:// doi.org/10.4269/ajtmh.15-0435

12. Paris DH, Dumler JS. State of the art of diagnosis of rickettsial diseases: the use of blood specimens for diagnosis of scrub typhus, spotted fever group rickettsiosis, and murine typhus. Curr Opin Infect Dis. 2016;29:433-9. https://doi.org/10.1097/QCO.0000000000000298

13. Yoo JR, Heo ST, Kang JH, Park D, Kim JS, Bae JH, et al. Mixed infection with severe fever with thrombocytopenia syndrome virus and two genotypes of scrub typhus in a patient, South Korea, 2017. Am J Trop Med Hyg. 2018;99:287-90. https://doi.org/10.4269/ajtmh.18-0088

14. Kim DM, Lee YM, Back JH, Yang TY, Lee JH, Song HJ, et al. A serosurvey of Orientia tsutsugamushi from patients with scrub typhus. Clin Microbiol Infect. 2010;16:447-51. https://doi.org/10.1111/j.1469-0691.2009.02865.x

15. Yun SM, Park SJ, Kim YI, Park SW, Yu MA, Kwon HI, et al. Genetic and pathogenic-diversity of severe fever with thrombocytopenia syndrome (SFTSV) in South Korea. JCI Insight. 2020;5:e129531.

Addresses for correspondence: Nam-Hyuk Cho, Seoul National

University College of Medicine, 103 Daehak-ro, Jongno-gu,

Seoul 03080, South Korea; email: chonh@snu.ac.kr;

Keun-Hwa Lee, Hanyang University College of Medicine,

Department of Microbiology, 222 Wangsimni-ro, Seongdong-gu,

Seoul 04763, South Korea; email: yomust7@gmail.com 